\title{
Differentiation of Mouse Primordial Germ Cells into Functional Oocytes In Vitro
}

\author{
Kanako Morohaku, ${ }^{1}$ Yuji Hirao, ${ }^{2}$ and Yayoi Obata ${ }^{1}$ \\ ${ }^{1}$ Department of Bioscience, Tokyo University of Agriculture, 1-1-1 Sakuragaoka, Setagaya-ku, Tokyo 156-8502, Japan; and \\ ${ }^{2}$ Institute of Livestock and Grassland Science, NARO, 2 Ikenodai, Tsukuba, Ibaraki 305-0901, Japan
}

(Received 17 November 2016; accepted 15 February 2017; published online 27 February 2017)

Associate Editor Christiani Amorim oversaw the review of this article.

\begin{abstract}
Various complex molecular events in oogenesis cannot be observed in vivo. As a bioengineering technique for female reproductive tissues, in vitro culture systems for female germ cells have been used to analyze oogenesis and preserve germ cells for over 20 years. Recently, we have established a new methodological approach for the culture of primordial germ cells (PGCs) and successfully obtained offspring. Our PGC culture system will be useful to clarify unresolved mechanisms of fertility and sterility from the beginning of mammalian oogenesis, before meiosis. This review summarizes the history of culture methods for mammalian germ cells, our current in vitro system, and future prospects for the culture of germ cells.
\end{abstract}

Keywords-Primordial germ cell, In vitro oocyte growth, Oocyte preservation.

\section{INTRODUCTION}

In mice, primordial germ cells (PGCs) first emerge at around 7.5 days post-coitum $(\mathrm{dpc}){ }^{27}$ They are defined by high levels of tissue-nonspecific alkaline phosphatase activity and/or as Dppa3/PGC7/stellapositive cells at the base of the allantois. ${ }^{83}$ PGCs are specified by Blimp1/Prdm1 and $\operatorname{Prdm} 14$ expression prior to $7.5 \mathrm{dpc} .{ }^{69,104}$ They migrate into gonads with the help of chemotaxis factors, such as c-kit/Kit and kit ligand/Kitl, ${ }^{15,26,110,111}$ until $10.5 \mathrm{dpc}$ and rapidly proliferate from approximately 40 to 25,000 in number between 7.5 and 13.5 dpc. $^{95}$ During this period, PGCs become progressively different from their ancestors; over time, they exhibit the repression of genes char-

Address correspondence to Yayoi Obata, Department of Bioscience, Tokyo University of Agriculture, 1-1-1 Sakuragaoka, Setagaya-ku, Tokyo 156-8502, Japan. Electronic mail: ylobata@nodai.ac.jp acteristic of their neighboring somatic cells, ${ }^{83}$ reprogramming including the erasure of genomic imprinting, ${ }^{41,61,88,89}$ and the acquisition of sexuality. $1,36,57,84$ Consequently, they are ready for oogenesis or spermatogenesis.

Oogenesis begins with the differentiation of isomorphic PGCs into oogonia following sexual differentiation. Mammalian PGCs and oogonia mitotically divide and reach a maximum number at the fetal stage (Table 1). ${ }^{5,9,11,29,34,48,49,54,58,64,79,106}$ In female mouse embryos, PGCs receive retinoic acid signals from the adjacent mesonephros and Stra8 expression is then induced. ${ }^{44,73}$ STRA8 requires pre-meiotic DNA replication. ${ }^{6}$ As a result, PGCs cease proliferation and enter meiosis at around $14.5 \mathrm{dpc}$ in females, but are arrested at $\mathrm{G} 1 / \mathrm{G} 0$ in the mitotic stage until a few days after birth in males. ${ }^{55}$ It has been thought that all oogonia are destined to enter meiosis in fetal ovaries, after which more than half of oocytes are lost by apoptosis. ${ }^{5,9,64}$ Surviving oocytes are assembled into primordial follicles. These primordial follicles become dormant and only a small proportion are activated to produce fully matured oocytes at the adult stage. The limited number of mature oocytes represents a disadvantage for breeding, reproduction, and scientific research. Furthermore, the regulatory mechanisms of mammalian oogenesis remain largely unknown.

In vitro systems have helped elucidate mechanisms underlying PGC specification, proliferation, and differentiation. Recently, we successfully demonstrated the complete in vitro generation of fertile mouse oocytes from PGCs for the first time. ${ }^{63}$ Such an in vitro system is expected to unravel the mechanisms of oogenesis and preserve female gametes. In this review, we describe the brief history of the in vitro systems for 
TABLE 1. The numbers of germ cells in mammalian species.

\begin{tabular}{|c|c|c|c|c|c|}
\hline \multirow[b]{2}{*}{ Species } & \multicolumn{2}{|c|}{$\begin{array}{l}\text { Estimated maximum total no. of germ cells in the } \\
\text { ovary }\end{array}$} & \multirow{2}{*}{$\begin{array}{l}\text { Estimated total no. } \\
\text { of germ cells in the } \\
\text { ovary at birth }\end{array}$} & \multirow{2}{*}{$\begin{array}{l}\text { Estimated total no. of } \\
\text { germ cells in the ovary } \\
\text { at puberty }\end{array}$} & \multirow[b]{2}{*}{ References } \\
\hline & Approx. no. of germ cells & Stage & & & \\
\hline Mouse (C57BL/6) & 15,000 & $15.5 \mathrm{dpc}$ & 7000 & $3000-5000$ & 11,64 \\
\hline Rat & 75,000 & $18.5 \mathrm{dpc}$ & 52,000 & $5000-10000$ & 9,49 \\
\hline Human & $5 \times 10^{6}-7 \times 10^{6}$ & Midgestation & $5 \times 10^{5}-1 \times 10^{6}$ & $1.5 \times 10^{5}-3 \times 10^{5}$ & $5,29,48$ \\
\hline Rhesus monkey & - & - & $4 \times 10^{5}$ & - & 34 \\
\hline Bovine & $2 \times 10^{6}$ & Prenatal & $1.2 \times 10^{5}-1.5 \times 10^{5}$ & - & 106 \\
\hline Sheep & $9 \times 10^{5}$ & Day 75 of gestation & - & $30,000-50,000$ & 79 \\
\hline Pig & $8 \times 10^{5}-1.2 \times 10^{6}$ & Day 90 of gestation & $4.5 \times 10^{5}$ & - & 58 \\
\hline
\end{tabular}

recapitulating germ cell development and summarize the development and current state of these cutting-edge techniques for PGC/oocyte culture. We also discuss potential future applications of our advanced technique, e.g., for large-scale oocyte production, identification of the requirements for fertile oocytes, and visualization of oogenesis.

\section{HISTORY OF PGC CULTURE IN MICE}

Early studies on germ cell culture focused on determining how mammalian PGCs migrate into gonads and subsequently differentiate into oocytes. In the 1980s, Tam and Snow removed small pieces of the primitive streak containing the future PGCs-fated region at 6.5 and $7.5 \mathrm{dpc}$ and cultured them in DMEM on plastic dishes owing to the difficulties in tracing PGC fate in vivo. The small pieces increased in size after $24 \mathrm{~h}$ of culture, but growth was arrested at $48 \mathrm{~h} .{ }^{95}$ McLaren and colleagues isolated PGCs from female gonads at $13.5 \mathrm{dpc}$, and tried to culture them in vitro without feeder cells. These PGCs survived and progressed into meiosis, suggesting that female PGCs at $13.5 \mathrm{dpc}$ are committed to enter meiosis, independent of the gonadal environment. ${ }^{17}$ Later, it was found that the culture of isolated PGCs on STO cells (a mouse embryonic fibroblastic cell line) effectively extends PGC survival and enables the successful recapitulation of PGC migration in vitro. ${ }^{19,93}$ STO cells produce various key factors for PGC proliferation, such as kit ligand (also known as stem cell factor (SCF) or steel factor) and leukemia inhibitory factor (LIF), at around 8.5-11.5 dpc. ${ }^{52}$ The importance of STO cells for PGC culture can be explained by the phenotypes and genotypes in $W / W$ and $S l / S l$ mutant mice, which are sterile because PGCs are incapable of migration into gonads and proliferation. It was found that the $W$ locus encodes c-kit/Kit, a receptor for the kit ligand, in 1988 and the Sl locus encodes kit ligands/Kitl, in 1990. ${ }^{15,26,110,111}$
Recent studies have concentrated on PGC specification. Yoshimizu et al. cultured epiblasts from 5.5dpc embryos with or without extra-embryonic tissues, demonstrating that PGC emergence requires extraembryonic tissues. ${ }^{107}$ PGC generation from proximal epiblasts requires BMP4 from extra-embryonic tissues. ${ }^{45}$ Breakthrough experiments performed by Saitou and colleagues have shown that PGC-like cells (PGCLCs) are successfully differentiated in vitro from epiblasts of 6.0-dpc embryos in which PGCs are not specified. ${ }^{68}$ They found that BMP4 and WNT3 are indispensable for the activation of Blimpl and Prdm14 in the posterior part of the proximal epiblast from which PGCs arise. WNT3 induces T(BRACHYURY) expression, leading to the activation of Blimpl and Prdm14. Both Blimp1 and Prdm14 are transcriptional repressors essential for the loss of somatic cell fate and PGC specification. ${ }^{3}$ After PGC specification, BMP4, BMP8b, LIF, Kit ligand, and EGF enhance the proliferation of PGCLCs in vitro. PGCLCs exhibit the erasure of genomic imprinting. Consequently, they develop into functional sperm following transplantation to beneath the tunica albuginea of adult testes. ${ }^{68}$ Interestingly, PGCs proliferate in vitro, but their growth is arrested at corresponding time points in vivo. ${ }^{19,28,52,68}$

In the presence of basic fibroblast growth factor (bFGF), Kit ligand, and LIF, PGCs are reprogrammed and acquire pluripotency and infinite proliferation activity. ${ }^{53,78,81}$ These cells are called embryonic germ (EG) cells and are no longer equal to PGCs. Recently, EG cells have also been established via the activation of serine/threonine kinase $\mathrm{AKT},{ }^{51}$ trichostatin A, histone deacetylase inhibitor, ${ }^{21}$ or inhibitors of mitogen-activated protein kinase signaling and of glycogen synthase kinase $3(2 \mathrm{i}) .^{47}$ In vitro systems to extend PGC proliferation while maintaining their intrinsic properties, have not been developed to date.

Culture methods for fetal gonads containing PGCs have also been established to examine the mechanisms 
of gonadal somatic cell and PGC differentiation. Until PGCs cease proliferation in vivo, gonadal somatic cells commit to the sexual differentiation of PGCs. Studies on the role of gonadal somatic cells in sexual differentiation have shown that the timing of meiotic progression in the indifferent gonads from $11.5-\mathrm{dpc}$ embryos is altered by culture with ovaries or testes from 14.5-dpc embryos on 1\% agar on a Nuclepore filter. ${ }^{12,94}$ Later, using a gas-liquid interface culture system in which gonads were cultured on a small block of $2 \%$ agar or on a micropore membrane filter with a thin layer of culture medium, more precise results were obtained. The culture of sexually chimeric gonads produced by the aggregation of XY gonadal somatic cells and XX germ cells or the opposite combination showed that the sex of germ cells is committed by gonadal somatic cells at $11.5-12.5 \mathrm{dpc}$ in males and $12.5-13.5 \mathrm{dpc}$ in females. ${ }^{1}$ This method also improved germ cell development, e.g., PGCs in the gonads obtained from 11.5-dpc female embryos were able to differentiate into oocytes with diameters of greater than $60 \mu \mathrm{m}$ after 23 days of culture.$^{56}$ However, organ culture systems have not been designed to enable the completion of oogenesis or spermatogenesis. In vitro gametogenesis does not exactly recapitulate events that occur during gametogenesis in vivo unless fertile gametes are produced. Eppig et al. successfully cultured newborn ovaries containing non-growing oocytes and the derivative secondary follicles to obtain mature oocytes, which were able to develop to term after in vitro fertilization. ${ }^{22}$ Accordingly, they established a system with the potential to precisely recapitulate oogenesis. Ogawa et al. also demonstrated the cultivation of neonatal testes containing prospermatogonia on agar, yielding fertile sperm after intracytoplasmic sperm injection. ${ }^{87}$ However, the entire process of either oogenesis or spermatogenesis from PGCs to mature gametes has not been replicated in vitro in the 20 years since these studies.

\section{COMPLETION OF MOUSE OOGENESIS IN VITRO}

The production of fertile oocytes from PGCs, oogonia, or immature oocytes provides a basis for understanding the mechanisms of oogenesis in coordination with folliculogenesis and for preserving female gametes. Ovarian somatic cells are essential for the in vitro recapitulation of oogenesis. ${ }^{39}$ Ovaries consist of granulosa cells, theca cells, oocytes, and stromal cells. They produce numerous cytokines and steroid hormones to support oogenesis and self-organization via paracrine and autocrine signaling. ${ }^{72}$ These factors have not been comprehensively identified; accordingly, the culture of ovaries and/or follicles has been adopted for establishing an in vitro system, rather than the culture of oocytes alone, without their surrounded follicle cells, in livestock, rodents, nonhuman primates and humans. ${ }^{4,24,39,92,96}$

Generally, the developmental ability of oocytes grown in vitro is limited by long culture times and a lack of appropriate culture conditions. To overcome these difficulties, ovarian pieces derived from fetuses or juveniles are transplanted into adult mice, resulting in the successful production of larger quantities of highquality oocytes from explanted grafts than are obtained in vitro. ${ }^{91}$ Several studies have shown that the xenogenetic transplantation of ovaries into immunodeficient mice induces oocyte growth. ${ }^{7,10,60,71}$ Thus, an ex vivo strategy may be beneficial when useful fetuses/ animals die prior to birth/puberty or for the recovery of fertility in ovariectomized cancer patients. Yet, an ex vivo strategy cannot be used to produce functional oocytes as effectively as intact ovaries, ${ }^{50}$ cannot completely prevent the reintroduction of cancer cells in patients, and is less appropriate for studies of oogenesis because it is blinded to sequential changes in oogenesis.

Ovarian/follicular culture has been examined extensively in several mammals. Meiotically mature oocytes are successfully developed by the culture of preantral follicles, oocyte-granulosa complexes, or ovarian pieces in human, bovine, sheep, and pig. ${ }^{8} 14,38,65,70,103$ However, in vitro systems have poor outcomes depending on the length of the culture period required for the completion of oogenesis. Among mammals, mouse oocytes with proven fertility have been successfully produced from early-stage oocytes at comparably high efficiency in vitro. ${ }^{31,59,62,66}$ Live mouse pups have been obtained from the culture of secondary follicles derived from ovaries of juveniles and from a 2-step culture of neonatal primordial follicles, i.e., ovarian culture followed by follicle culture. $22,23,31,62,66$

Compared to the culture of immature oocytes embedded in the primordial or secondary follicles, a greater number of events in oogenesis need to be achieved in PGC culture. ${ }^{4,24,92,96}$ For example, prior to switching from mitosis to meiosis, female germ cells form cysts via incomplete cytokinesis. Oocytes cysts are broken after oocytes enter meiosis, and each oocyte is enclosed by a few flattened granulosa cells to form a primordial follicle; the first meiosis is then arrested at the diplotene stage of prophase I. Many studies have attributed female sterility to abnormalities in meiosis or follicle formation. ${ }^{85} \mathrm{~A}$ complete in vitro system for recapitulating oogenesis endows oocytes with totipotency and fertility. However, existing methods are not sufficient to reproduce oogenesis. The resultant oo- 
cytes do not reach the second meiosis or do not acquire ooplasmic competency to support full-term development. ${ }^{18,67,90,109}$

One issue is the long duration required for organ culture to produce fertile oocytes, i.e., 4 weeks or more. The ovaries/gonads are separated from the vasculature to supply nutrition and hormones from the mother, placenta, and neighboring/distal organs via endocrine systems and to support gas exchange. ${ }^{17,56,109}$ This causes low metabolic activity, degradation of supporting cells, and low-quality oocytes. Hence, the culture system needs to be switched to follicle culture from organ culture after each oocyte is enclosed by follicular cells. However, applying a 2-step culture system established for neonatal ovaries to fetal gonads containing PGCs has not been achieved. Conventional culture conditions cause hypoplasia of follicles in the gonads. Consequently, follicles cannot be isolated from cultured ovaries when the starting point for organ culture is prior to follicle formation in vivo. ${ }^{67,91,109}$ Even though many oocytes grow in size without being enclosed by a follicle structure, they never reach the functionally mature stage. This is a major limitation in the production of fertile oocytes from PGCs in vitro. We previously showed that nuclear transfer between in vivo-derived fully grown oocytes and in vitro-derived immature oocytes is needed to overcome the incompetence of oocytes differentiated from PGCs in vitro. Some reconstituted oocytes develop to offspring. ${ }^{67}$ However, a true in vitro system is required because nuclear transfer experiments mask the essential factors for the acquisition of oocyte competence and the mechanisms by which oocytes acquire competence, similar to ex vivo strategies.

A breakthrough in PGC culture has come from the findings of Pepling and our studies. ${ }^{16,63}$ We adapted an ordinary 2-step culture system, which was established by Eppig and colleagues, to grow oocytes in newborn mouse ovaries for PGC culture ${ }^{22,66}$ (Fig. 1). Mouse embryonic gonads from 12.5-dpc embryos were cultured for 17 days on a Transwell-COL membrane within a thin layer of culture medium containing $10 \%$ fetal bovine serum (FBS). The number of isolated secondary follicles per ovary on day 17 of the culture was low (average, 6.2 follicles per ovary), ${ }^{63}$ consistent with previous results. ${ }^{67,90,109} \mathrm{~A}$ histological analysis of cultured ovaries showed multiple-oocyte follicles and the failure of each oocyte to be enclosed in the follicle. These results indicated abnormal follicle formation and explained the low yield of secondary follicles from embryonic ovaries (Fig. 2). To improve the failure of follicle development in the culture, we focused on oocyte cyst breakdown that occurs in the middle of the culture period. We surmised that the cytokinesis of oocytes is not completed or is delayed in vitro. Oocyte cyst breakdown occurs at or just prior to the time when a single oocyte is surrounded by granulosa cells. ${ }^{76} \mathrm{In}$ previous reports by Pepling and colleagues, the introduction of estrogen into the organ culture medium prevented oocyte cyst breakdown in newborn mouse ovaries. ${ }^{16}$ Some reports have suggested an association between follicle formation in fetal ovaries and a decrease in estrogen in vivo. ${ }^{105}$ However, maternal- or placenta-derived estrogen is completely isolated by transferring fetal gonads to the in vitro environment. Therefore, to understand the molecular basis for abnormalities in follicle formation in vitro, we conducted RNA sequencing (RNA-seq) in fetal-derived ovaries after 7 days of culture and compared transcripts with those of neonatal ovaries on the corresponding day. An RNA-seq analysis showed that more than 500 genes are differentially expressed in in vitro-derived ovaries compared with neonatal ovaries. Interestingly, the most common upstream regulator of these differentially expressed genes was estrogen. Estrogen binds to estrogen receptor 1 (ESR1), estrogen receptor 2 (ESR2), and G protein-coupled estrogen 1 (GPER1). ESR 1 and ESR2 in the presence of bound estrogen bind to estrogen response elements (5'AGGTCAnnnTGACCT-3') and regulate transcription. ${ }^{43}$ There was no evidence of substantial amounts of estrogen in the medium or that ESR1 and ESR2 were elevated in the in vitro-derived ovaries. Therefore, we hypothesized that 1) FBS contains estrogen-like factor(s) that can bind to ESR1 and/or ESR2, or 2) FBS contains many materials (e.g., cholesterol) needed to synthesize estrogen within the ovaries. To test these hypotheses, gonads from embryos at $12.5 \mathrm{dpc}$ were cultured in medium supplemented with FBS for 17 days. From day 5 to day 11 when oocyte cyst breakdown occurs, an antagonist of both ESR1 and ESR2, ICI 182,780 (ICI), was added, an inhibitor of the aromatase, anastrozole, was added (unpublished data), or serum protein substitute (SPS) was added instead of FBS (Fig. 1). ICI 182,780 is known as fulvestrant and is used for breast cancer therapy to minimize estrogen activity. ${ }^{82,97}$ Anastrozole is also used for breast cancer therapy to inhibit estrogen production. The number of isolated secondary follicles was dramatically higher in the ICI-treated group and moderately higher in the SPS group compared to that in the FBS group (average, 82.0 follicles per ovary for $10 \mu \mathrm{M}$ ICI, 27.2 follicles per ovary for SPS, and 6.2 follicles in the FBS group). Immunohistochemical analysis showed that each oocyte was enclosed within the follicle with two or more layers of granulosa cells in the ICI and SPS groups (Fig. 2). Since anastrozole had no effect on the number of isolated secondary follicles (average, 2.3 follicles per ovary, unpublished data) and their phenotypes, ovaries would not produce excessive 




FIGURE 1. Timeline for PGC culture. Our culture system for PGCs is consisted of a gonadal culture and a follicle culture, and takes a month to obtain matured oocytes from 12.5-dpc embryonic gonads. We examined several culture conditions from day 5 to day 11: Gonads from embryos at 12.5-dpc embryos were cultured in 10\% FBS-containing alpha MEM (FBS group), cultured in 10\% FBS- and 1-10 $\mu \mathrm{M} \mathrm{ICl-containing} \mathrm{alpha} \mathrm{MEM} \mathrm{(ICl} \mathrm{group),} \mathrm{cultured} \mathrm{in} \mathrm{10 \%} \mathrm{SPS-containing} \mathrm{alpha} \mathrm{MEM} \mathrm{instead} \mathrm{of} \mathrm{FBS} \mathrm{(SPS} \mathrm{group),}$ and cultured in 10\% FBS- and 1-50 $\mu \mathrm{M}$ anastrozole-containing alpha MEM (anastrozole group). ICI group was the best culture condition to produce secondary follicles in vitro. Secondary follicles appeared in ovaries in vitro by day 17 of culture and were then isolated from ovaries mechanically for the follicle culture. At day 20 , the follicles were treated with $0.1 \%$ collagenase, thereafter, they were cultured for another 9-11 days.

estrogen in vitro. Furthermore, the addition of estradiol to the medium containing ICI or SPS complementarily decreased the number of secondary follicles per ovary. Therefore, we concluded that the upregulation of estrogen signaling resulted in abnormal secondary follicle development in vitro and ICI addition to the medium for gonadal culture overcame this abnormality.

We also modified the follicle culture protocol established by Eppig in $1989 .{ }^{23}$ Our previous study showed that polyvinylpyrrolidone (PVP), a highmolecular-mass compound, improved follicle growth and survival in vitro in both bovines and mice. ${ }^{37,62}$ Therefore, we added $2 \%$ PVP to the medium for the follicle culture (Fig. 1). We observed a more striking impact of PVP on the follicles isolated from in vitro-derived ovaries than on those from in vivo-derived ovaries. The survival rate increased by at least 3 times by the addition of PVP to the medium. It is not clear why PVP is effective for increasing the 

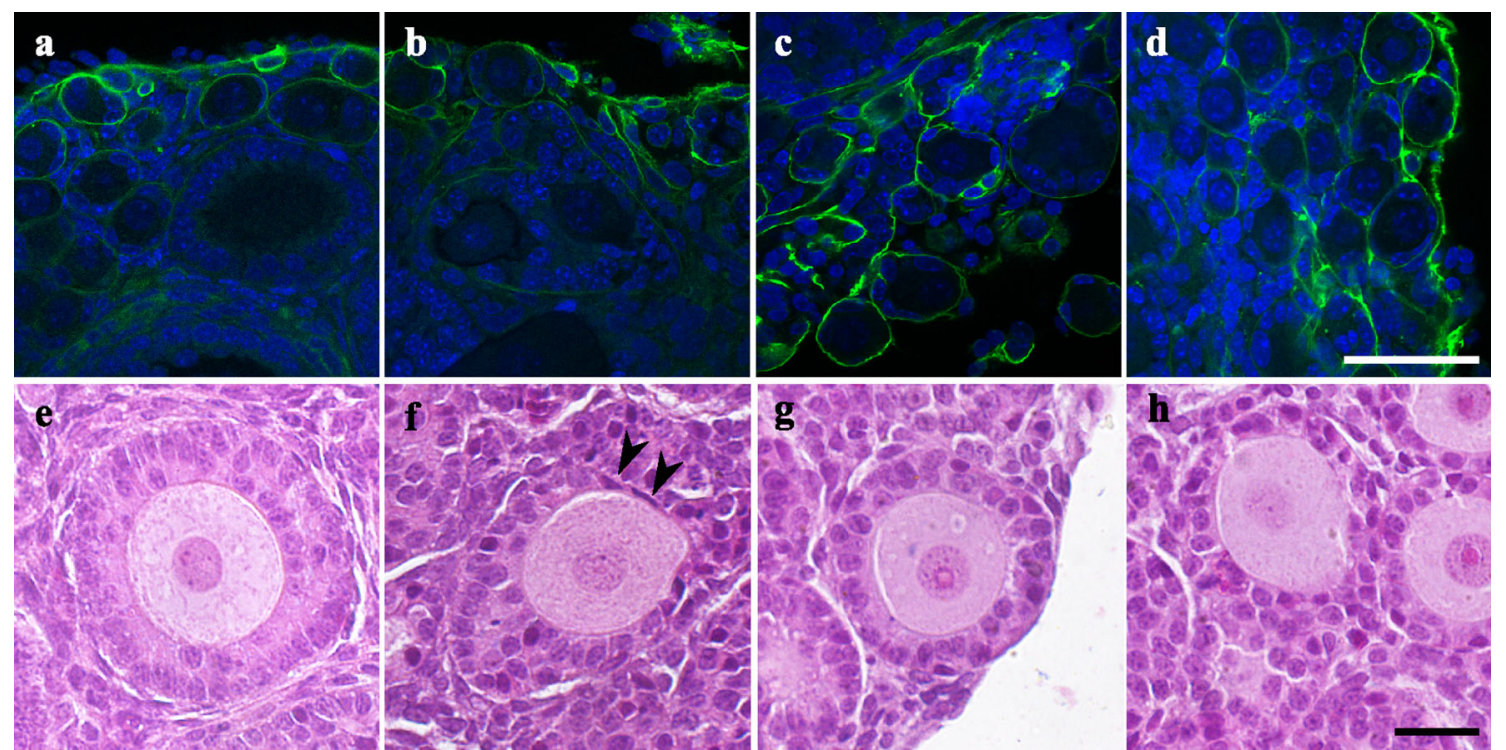

FIGURE 2. Morphology of in vitro grown follicles. (a-d) Immunofluorescence staining of the extracellular matrix. Ovaries derived from 10-dpn mouse (a), FBS group (b), ICl group (c), and SPS group (d). Each follicle was enclosed by laminin in both ICl and SPS groups but not in FBS group. Green, laminin; Blue, nuclei. (e-g) Histology sections of ovaries. Secondary follicles in the ovary of 10-dpn mouse (e), FBS group (f), and ICl group ( $g, h)$. Alignment of follicular cells was less regular in the ovaries of FBS group. Flattened theca like-cells attached to oocyte (black arrowheads) in FBS group (f). ICI group, secondary follicles were clearly formed (g), but the borders of some follicles were not clear (h). Bar $=50 \mu \mathrm{m}$.

survivability and growth of follicles. However, dextran, a representative macromolecular substance, has been used for organ preservation. We speculate that PVP might play a role in sustaining the structure of oocyte-surrounding follicle cells, maintaining their viability and preventing the diffusion of cytokines into the medium. In fact, PVP increased the mRNA expression of genes encoding cytokines, such as BMP6, BMP15, c-kit, and kit ligand, in follicles during culture. $^{63}$

Another key to producing fertile oocytes from PGCs in vitro is collagenase treatment (Fig. 1). When we isolate secondary follicles from juvenile mice, ovaries are generally treated with collagenase. ${ }^{23}$ However, relatively fewer follicles were isolated after the collagenase treatment of in vitro-derived ovaries. This is attributed to the fragility of follicles from in vitro-derived ovaries and the random cellular alignment of some (Fig. 2f). We mechanically isolated secondary follicles, using a fine tungsten needle, and cultured intact follicles in medium containing $2 \%$ PVP. Follicles were able to grow; however, at the end of follicle culture, the layer of cumulus cells surrounding the oocyte was thin. The resultant oocyte could not develop beyond the 2-cell stage after fertilization. ${ }^{63}$ In contrast, collagenase treatment of mechanically isolated follicles exposed oocyte-granulosa cell complexes to the medium, resulting in an appropriate thickness of the cumulus cell layer surrounding the oocyte after follicle culture. The exposure of oocyte-granulosa cell complexes to the medium might promote gas exchange, nutritional intake, and the clearing of waste products via granulosa cells. The oocytes differentiated from PGCs in vitro grew to full size (approximately $80 \mu \mathrm{m}$ in diameter). Oocytes produced by this method exhibit successful fertilization, the completion of meiosis, and the acquisition of totipotency. Following the transplantation of 2-cell embryos in the oviducts of pseudopregnant mice, two to three pups per cultured gonad were born using our culture system. Pups from in vitro differentiated oocytes exhibited normal phenotypes and fertility. Oocyte-derived imprinting also persisted in these pups. ${ }^{63}$ Thus, a culture system for recapitulating oogenesis has been developed in a stepby-step manner.

\section{WIDELY APPLICABLE STRATEGY TO PRODUCE FERTILE OOCYTES FROM PGCS}

Vitrification is a useful technique for germ cell preservation. It is a kind of cryopreservation that avoids ice crystal formation by passing the cryohydric point quickly and therefore minimizes less cell damage. ${ }^{80}$ Vitrification as an alternative method for cryopreservation has been used for the preservation of oocytes, zygotes, and ovarian tissues in mice, bovines, humans, and so on. ${ }^{2}$ In our previous reports, functional oocytes and pups were successfully obtained from gonads vitrified/warmed following the method 
reported by Wang et al..$^{99}$ In brief, ovaries derived from 12.5 -dpc embryos were equilibrated for $20 \mathrm{~min}$ in vitrification medium containing $10 \%$ ethylene glycol, $10 \%$ dimethylsulfoxide (DMSO), and $4 \mathrm{mg} / \mathrm{ml}$ bovine serum albumin (BSA) in L15 base medium, and then for three minutes in $17 \%$ ethylene glycol, $17 \%$ DMSO, $0.75 \mathrm{M}$ sucrose, and $4 \mathrm{mg} / \mathrm{ml} \mathrm{BSA}$. After equilibration, the gonads were transferred to a cryotube and vitrified at $-196{ }^{\circ} \mathrm{C}$ in liquid nitrogen. A warming procedure was carried out in $0.5 \mathrm{M}$ sucrose for $3 \mathrm{~min}$ at $37^{\circ} \mathrm{C}$, and for $2 \mathrm{~min}$ at room temperature. Then, the gonads were washed in $0.25 \mathrm{M}$ sucrose, $0.125 \mathrm{M}$ sucrose, and culture medium, in sequence. Following warming, the gonads were cultured using our abovedescribed methods with ICI incorporated in the organ culture medium, PVP in the follicle culture medium, and collagenase treatment (Fig. 3). Although the efficiency by which secondary follicles were obtained was low compared with that for non-vitrified gonads, we successfully obtained pups from the culture of vitrified/ warmed ovaries.

\section{FUTURE PERSPECTIVES FOR PGC CULTURE}

Many key factors determining the growth of oocytes and follicles have been identified, including kit ligand, GDF9, BMP4, BMP7, activin, inhibin, EGF, FSH, and so on. ${ }^{62}$ Granulosa and theca cells support oocyte growth by secreting factors, and oocytes also produce factors for follicle cell differentiation and proliferation. In our system, the medium contains FBS during the whole culture period prior to fertilization. We used alpha-MEM supplemented with ascorbic acid and ICI for gonadal culture, and ascorbic acid, PVP, and FSH for follicle culture. When $10 \%$ SPS, which consists of serum albumin and alpha, beta, and gamma globulins, was used for gonadal culture instead of $10 \%$ FBS throughout organ culture, gonad growth was restricted and only low-quality follicles with a thin layer of granulosa cells formed. Knockout serum replacement had the same effect or was inferior to SPS supplementation. The duration of the development of fertile oocytes from PGCs is very long, but oocytes do not undergo renewal. Therefore, the accumulation of tiny defects leads to a loss of fertility in oocytes. To establish an in vitro system for recapitulating oogenesis, for the first time, FBS cannot be excluded from the medium.

Eppig et al. established a chemically defined medium for follicle culture. It contains BSA, insulin, transferrin, selenium, FSH, EGF, and fetuin, ${ }^{22}$ and has been adopted for human follicle culture, with some modifications. ${ }^{103}$ In 1996, for the first time, the successful growth of oocytes capable of developing to offspring from neonatal ovaries was demonstrated following a 2-step culture, i.e., ovarian culture with FBS-containing medium for 8 days and follicle culture with chemically defined medium for 14 days, as described above. Although this was a substantial achievement, the culture of gonads or ovaries still requires FBS. Moreover, oocytes produced from neonatal ovaries by 2-step culture with FBS-containing medium during the whole period have a greater potential to develop to term than those produced by Eppig et al. ${ }^{22,62,66}$ It is possible that various growth factors supplied by FBS are necessary in the culture medium. However, chemically defined medium is essential for increasing our understanding of the mechanisms of oogenesis.

In recent studies, PGCLCs have been differentiated from mouse embryonic stem cells and induced pluripotent stem (iPS) cells $\mathrm{s}^{32}$ and successfully developed to fertile oocytes in vitro. ${ }^{35}$ Accordingly, the complete reconstitution of the process from nongermline cells to female germ cells can be accomplished in vitro. Since fertile oocytes can be produced from mitotically divided cells, such an in vitro system would
Day 0

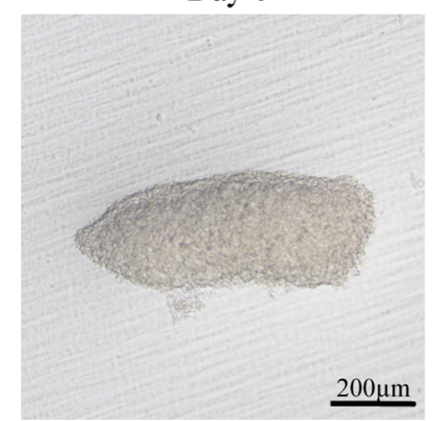

Day 17

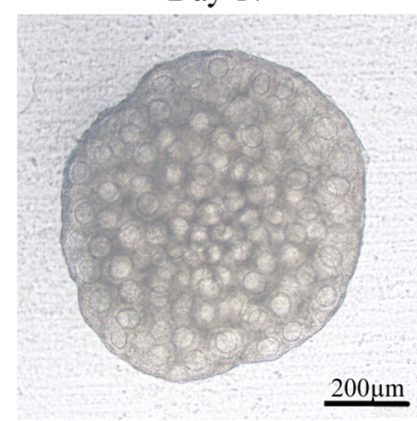

Isolation

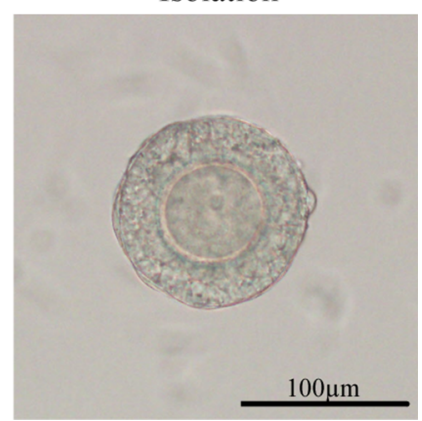

FIGURE 3. Vitrified-warmed gonads for production of oocytes. The gonad was cut into two or three pieces, dipped in the vitrification solution and frozen in liquid nitrogen $\left(\mathrm{LN}_{2}\right)$. Bright-field images show thawed gonads cultured for 0 and 17 days. Bar $=200 \mu \mathrm{m}$. A representative isolated follicle is labelled "isolation." Bar $=100 \mu \mathrm{m}$. 
expand the possibilities of the mass production of mammalian oocytes, sequential observations of oogenesis, and gene modifications during oogenesis using genome editing, RNA interference, or transfection technologies.

A culture system for PGCs has the potential to be applied to livestock and other mammals, but PGCs in these taxa are not well-characterized compared to those of mice. In pigs, the differentiation of PGCLCs to spermatogonial stem cell-like cells, but not spermatozoa, was observed after injection into busulfantreated mouse testes. ${ }^{101}$ It may be possible to obtain functional oocytes in large animals from PGCs or PGCLCs; however, optimal culture conditions, including ICI addition and its concentration, should be examined in each species and at each age. Even in mice, there is variation in hormone levels and the timing of primordial follicle formation among strains. ${ }^{77}$ In bovines, estradiol has inhibitory effects on primordial follicle assembly, ${ }^{105}$ but promotes follicle formation in hamsters and baboons. ${ }^{98,100}$ Thus, although a prototype in vitro system to produce functional oocytes from PGCs and PGCLCs has been established in mice, further investigation is required for establish a system that is widely applicable across taxa.

The introduction of our in vitro system to human PGC culture is impractical. Human PGCs differentiate into oocytes by 2 months post-conception, and primordial follicle formation starts by 6 months postconception $^{5}$; therefore, there are no PGCs in adult ovaries. Recently, PGCLCs have been established from human iPS cells supplemented with BMP4, LIF, $\mathrm{SCF}$, and EGF. ${ }^{86}$ However, differentiation of oocytes and spermatozoa from PGCLCs currently requires aggregation with somatic cells from embryonic gonads, at least in mice. ${ }^{32,33,35}$ If possible, it might take far longer to produce mature oocytes from human PGCs/ PGCLCs. Even in follicle culture, it takes over 30 days to grow small antral follicles from secondary follicles in vitro, ${ }^{96}$ and there is no evidence for the development of human preantral follicles beyond Graafian follicles in vitro. At all steps, the culture of human PGCs/ PGCLCs to produce mature oocytes raises ethical issues and safety concerns that have not been addressed.

For the last two decades, the existence of oogonial stem cells (OSCs) in adult ovaries has been a controversial topic. This idea stems from the discrepancy between the estimated number of oocytes in neonatal ovaries and the estimated number of ovulated oocytes and atretic follicles. Johnson et al. indicated that faster depletion of oocytes in the ovaries would be caused by a higher number of atretic follicles and ovulated oocytes if neo-oogenesis does not progress to adulthood. ${ }^{40} \mathrm{An}$ increasing number of reports has supported the existence of OSCs in the adult ovaries of mice, rats, bovi- nes, and humans. ${ }^{20,30,40,46,74}$ In these reports, OSCs are collected from adult ovaries by live-cell sorting using fluorescent- or magnetic-activated cell sorting (FACS or MACS) with germ cell or stem cell markers, such as MVH (known as Ddx4). ${ }^{102}$ Although the ratio of sorted OSCs after FACS or MACS was low in these studies, OSCs proliferated with the expression of both germ cell and stem cell markers during culture, and contributed to oocytes after grafting in ovaries ex vivo. However, the use of an MVH antibody in a live-cell sorting assay to detect antigens on the cell surface is questionable because MVH is a germline-specific RNA helicase and is believed to exist in the cytoplasm. ${ }^{13,25,108}$ Several attempts to resolve this issue with the MVH antibody approach have been reported using an SSEA1 antibody or Ddx4-cre transgenic mice. ${ }^{42,75}$ However, it is still not clear whether OSCs exist. Our in vitro system could be used to evaluate whether OSCs exist to supply new oocytes to adult ovaries without transplantation of OSCs.

Thus, we demonstrated the fertility of mouse oocytes produced from PGCs in vitro. This new methodological approach has important implications for female germ cell preservation and studies of every process involved in mammalian oogenesis.

\section{ACKNOWLEDGMENTS}

This work was supported by Grants-in-Aid for Scientific Research 26450449 and 25114008 to Y.O.

\section{CONFLICT OF INTEREST}

There are no conflicts of interest to declare.

\section{OPEN ACCESS}

This article is distributed under the terms of the Creative Commons Attribution 4.0 International License (http://creativecommons.org/licenses/by/4.0/), which permits unrestricted use, distribution, and reproduction in any medium, provided you give appropriate credit to the original author(s) and the source, provide a link to the Creative Commons license, and indicate if changes were made.

\section{REFERENCES}

\footnotetext{
${ }^{1}$ Adams, I. R., and A. McLaren. Sexually dimorphic development of mouse primordial germ cells: switching from oogenesis to spermatogenesis. Development 129:1155-1164, 2002.
} 
${ }^{2}$ Amorim, C. A., M. Curaba, A. Van Langendonckt, M. M. Dolmans, and J. Donnez. Vitrification as an alternative means of cryopreserving ovarian tissue. Reprod. Biomed. online 23:160-186, 2011.

${ }^{3}$ Aramaki, S., K. Hayashi, K. Kurimoto, H. Ohta, Y. Yabuta, et al. A mesodermal factor, T, specifies mouse germ cell fate by directly activating germline determinants. Dev. Cell 27:516-529, 2013.

${ }^{4}$ Araujo, V. R., M. O. Gastal, J. R. Figueiredo, and E. L. Gastal. In vitro culture of bovine preantral follicles: a review. Reprod. Biol. Endocrinol. 12:78, 2014.

${ }^{5}$ Baker, T. G. A quantitative and cytological study of germ cells in human ovaries. Proc. R. Soc. Lond. Ser. B 158:417-433, 1963.

${ }^{6}$ Baltus, A. E., D. B. Menke, Y. C. Hu, M. L. Goodheart, A. E. Carpenter, et al. In germ cells of mouse embryonic ovaries, the decision to enter meiosis precedes premeiotic DNA replication. Nat. Genet. 38:1430-1434, 2006.

${ }^{7}$ Bao, R. M., E. Yamasaka, M. Moniruzzaman, A. Hamawaki, M. Yoshikawa, and T. Miyano. Development of vitrified bovine secondary and primordial follicles in xenografts. Theriogenology 74:817-827, 2010.

${ }^{8}$ Barboni, B., V. Russo, S. Cecconi, V. Curini, A. Colosimo, et al. In vitro grown sheep preantral follicles yield oocytes with normal nuclear-epigenetic maturation. PLoS ONE 6:e27550, 2011.

${ }^{9}$ Beaumont, H. M., and A. M. Mandl. A quantitative and cytological study of oogonia and oocytes in the foetal and neonatal rat. Proc. R. Soc. Lond. Ser. B 155:557-579, 1962.

${ }^{10}$ Bosch, P., H. J. Hernandez-Fonseca, D. M. Miller, J. D. Wininger, J. B. Massey, et al. Development of antral follicles in cryopreserved cat ovarian tissue transplanted to immunodeficient mice. Theriogenology 61:581-594, 2004.

${ }^{11}$ Bristol-Gould, S. K., P. K. Kreeger, C. G. Selkirk, S. M. Kilen, R. W. Cook, et al. Postnatal regulation of germ cells by activin: the establishment of the initial follicle pool. Dev. Biol. 298:132-148, 2006.

${ }^{12}$ Byskov, A. G., and L. Saxen. Induction of meiosis in fetal mouse testis in vitro. Dev. Biol. 52:193-200, 1976.

${ }^{13}$ Castrillon, D. H., B. J. Quade, T. Y. Wang, C. Quigley, and C. P. Crum. The human VASA gene is specifically expressed in the germ cell lineage. Proc. Natl. Acad. Sci. U.S.A. 97:9585-9590, 2000.

${ }^{14}$ Cecconi, S., B. Barboni, M. Coccia, and M. Mattioli. In vitro development of sheep preantral follicles. Biol. Reprod. 60:594-601, 1999.

${ }^{15}$ Chabot, B., D. A. Stephenson, V. M. Chapman, P. Besmer, and A. Bernstein. The proto-oncogene c-kit encoding a transmembrane tyrosine kinase receptor maps to the mouse W locus. Nature 335:88-89, 1988.

${ }^{16}$ Chen, Y., K. Breen, and M. E. Pepling. Estrogen can signal through multiple pathways to regulate oocyte cyst breakdown and primordial follicle assembly in the neonatal mouse ovary. J. Endocrinol. 202:407-417, 2009.

${ }^{17}$ De Felici, M., and A. McLaren. In vitro culture of mouse primordial germ cells. Exp. Cell Res. 144:417-427, 1983.

${ }^{18}$ Dong, H. S., L. Li, Z. H. Song, J. Tang, B. Xu, et al. Premeiotic fetal murine germ cells cultured in vitro form typical oocyte-like cells but do not progress through meiosis. Theriogenology 72:219-231, 2009.

${ }^{19}$ Donovan, P. J., D. Stott, L. A. Cairns, J. Heasman, and C. C. Wylie. Migratory and postmigratory mouse pri- mordial germ cells behave differently in culture. Cell 44:831-838, 1986.

${ }^{20}$ Dunlop, C. E., E. E. Telfer, and R. A. Anderson. Ovarian germline stem cells. Stem Cell Res. Ther. 5:98, 2014.

${ }^{21}$ Durcova-Hills, G., F. Tang, G. Doody, R. Tooze, and M. A. Surani. Reprogramming primordial germ cells into pluripotent stem cells. PLOS ONE 3:e3531, 2008.

${ }^{22}$ Eppig, J. J., and M. J. O'Brien. Development in vitro of mouse oocytes from primordial follicles. Biol. Reprod. 54:197-207, 1996.

${ }^{23}$ Eppig, J. J., and A. C. Schroeder. Capacity of mouse oocytes from preantral follicles to undergo embryogenesis and development to live young after growth, maturation, and fertilization in vitro. Biol. Reprod. 41:268-276, 1989.

${ }^{24}$ Fortune, J. E. The early stages of follicular development: activation of primordial follicles and growth of preantral follicles. Anim. Reprod. Sci. 78:135-163, 2003.

${ }^{25}$ Fujiwara, Y., T. Komiya, H. Kawabata, M. Sato, H. Fujimoto, et al. Isolation of a DEAD-family protein gene that encodes a murine homolog of Drosophila vasa and its specific expression in germ cell lineage. Proc. Natl. Acad. Sci. U.S.A. 91:12258-12262, 1994.

${ }^{26}$ Geissler, E. N., M. A. Ryan, and D. E. Housman. The dominant-white spotting (W) locus of the mouse encodes the c-kit proto-oncogene. Cell 55:185-192, 1988.

${ }^{27}$ Ginsburg, M., M. H. Snow, and A. McLaren. Primordial germ cells in the mouse embryo during gastrulation. Development 110:521-528, 1990.

${ }^{28}$ Godin, I., C. Wylie, and J. Heasman. Genital ridges exert long-range effects on mouse primordial germ cell numbers and direction of migration in culture. Development 108:357-363, 1990.

${ }^{29}$ Gougeon, A., and G. B. Chainy. Morphometric studies of small follicles in ovaries of women at different ages. $J$. Reprod. Fertil. 81:433-442, 1987.

${ }^{30}$ Grieve, K. M., M. McLaughlin, C. E. Dunlop, E. E. Telfer, and R. A. Anderson. The controversial existence and functional potential of oogonial stem cells. Maturitas 82:278-281, 2015.

${ }^{31}$ Hasegawa, A., N. Mochida, T. Ogasawara, and K. Koyama. Pup birth from mouse oocytes in preantral follicles derived from vitrified and warmed ovaries followed by in vitro growth, in vitro maturation, and in vitro fertilization. Fertil. Steril. 86:1182-1192, 2006.

${ }^{32}$ Hayashi, K., S. Ogushi, K. Kurimoto, S. Shimamoto, H. Ohta, and M. Saitou. Offspring from oocytes derived from in vitro primordial germ cell-like cells in mice. Science 338:971-975, 2012.

${ }^{33}$ Hayashi, K., H. Ohta, K. Kurimoto, S. Aramaki, and M. Saitou. Reconstitution of the mouse germ cell specification pathway in culture by pluripotent stem cells. Cell 146:519-532, 2011.

${ }^{34}$ Healy, D. L., J. Bacher, and G. D. Hodgen. Thymic regulation of primate fetal ovarian-adrenal differentiation. Biol. Reprod. 32:1127-1133, 1985.

${ }^{35}$ Hikabe, O., N. Hamazaki, G. Nagamatsu, Y. Obata, Y. Hirao, et al. Reconstitution in vitro of the entire cycle of the mouse female germ line. Nature 539:299-303, 2016.

${ }^{36}$ Hilscher, B., W. Hilscher, B. Bulthoff-Ohnolz, U. Kramer, A. Birke, et al. Kinetics of gametogenesis. I. Comparative histological and autoradiographic studies of oocytes and transitional prospermatogonia during oogenesis and prespermatogenesis. Cell Tissue Res. 154:443$470,1974$. 
${ }^{37}$ Hirao, Y., T. Itoh, M. Shimizu, K. Iga, K. Aoyagi, et al. In vitro growth and development of bovine oocyte-granulosa cell complexes on the flat substratum: effects of high polyvinylpyrrolidone concentration in culture medium. Biol. Reprod. 70:83-91, 2004.

${ }^{38}$ Hirao, Y., T. Somfai, and K. Naruse. In vitro growth and maturation of vitrified-warmed bovine oocytes collected from early antral follicles. J. Reprod. Dev. 60:68-72, 2014.

${ }^{39}$ Honda, A., M. Hirose, K. Inoue, H. Hiura, H. Miki, et al. Large-scale production of growing oocytes in vitro from neonatal mouse ovaries. Int. J. Dev. Biol. 53:605-613, 2009.

${ }^{40}$ Johnson, J., J. Canning, T. Kaneko, J. K. Pru, and J. L. Tilly. Germline stem cells and follicular renewal in the postnatal mammalian ovary. Nature 428:145-150, 2004.

${ }^{41}$ Kafri, T., M. Ariel, M. Brandeis, R. Shemer, L. Urven, et al. Developmental pattern of gene-specific DNA methylation in the mouse embryo and germ line. Genes Dev. 6:705-714, 1992.

${ }^{42}$ Khosravi-Farsani, S., F. Amidi, M. Habibi Roudkenar, and A. Sobhani. Isolation and enrichment of mouse female germ line stem cells. Cell J. 16:406-415, 2015.

${ }^{43}$ Klein-Hitpass, L., M. Schorpp, U. Wagner, and G. U. Ryffel. An estrogen-responsive element derived from the $5^{\prime}$ flanking region of the Xenopus vitellogenin A2 gene functions in transfected human cells. Cell 46:1053-1061, 1986.

${ }^{44}$ Koubova, J., D. B. Menke, Q. Zhou, B. Capel, M. D. Griswold, and D. C. Page. Retinoic acid regulates sexspecific timing of meiotic initiation in mice. Proc. Natl. Acad. Sci. U.S.A. 103:2474-2479, 2006.

${ }^{45}$ Lawson, K. A., N. R. Dunn, B. A. Roelen, L. M. Zeinstra, A. M. Davis, et al. Bmp4 is required for the generation of primordial germ cells in the mouse embryo. Genes Dev. 13:424-436, 1999.

${ }^{46}$ Lee, Y. M., T. H. Kim, J. H. Lee, W. J. Lee, R. H. Jeon, et al. Overexpression of Oct4 in porcine ovarian stem/ stromal cells enhances differentiation of oocyte-like cells in vitro and ovarian follicular formation in vivo. $J$. Ovarian Res. 9:24, 2016.

${ }^{47}$ Leitch, H. G., K. Blair, W. Mansfield, H. Ayetey, P. Humphreys, et al. Embryonic germ cells from mice and rats exhibit properties consistent with a generic pluripotent ground state. Development 137:2279-2287, 2010.

${ }^{48}$ Mamsen, L. S., M. C. Lutterodt, E. W. Andersen, A. G. Byskov, and C. Y. Andersen. Germ cell numbers in human embryonic and fetal gonads during the first two trimesters of pregnancy: analysis of six published studies. Hum. Reprod. 26:2140-2145, 2011.

${ }^{49}$ Mandl, A. M. A quantitative study of the sensitivity of oocytes to x-irradiation. Proc. R. Soc. Lond. Ser. B 150:53-71, 1959.

${ }^{50}$ Matoba, S., and A. Ogura. Generation of functional oocytes and spermatids from fetal primordial germ cells after ectopic transplantation in adult mice. Biol. Reprod. 84:631-638, 2011.

${ }^{51}$ Matsui, Y., A. Takehara, Y. Tokitake, M. Ikeda, Y. Obara, et al. The majority of early primordial germ cells acquire pluripotency by AKT activation. Development 141:4457-4467, 2014.

${ }^{52}$ Matsui, Y., D. Toksoz, S. Nishikawa, S. Nishikawa, D. Williams, et al. Effect of Steel factor and leukaemia inhibitory factor on murine primordial germ cells in culture. Nature 353:750-752, 1991.
${ }^{53}$ Matsui, Y., K. Zsebo, and B. L. Hogan. Derivation of pluripotential embryonic stem cells from murine primordial germ cells in culture. Cell 70:841-847, 1992.

${ }^{54}$ McCoard, S. A., T. H. Wise, and J. J. Ford. Germ cell development in Meishan and White Composite gilts. Anim. Reprod. Sci. 77:85-105, 2003.

${ }^{55}$ McLaren, A. Meiosis and differentiation of mouse germ cells. Symp. Soc. Exp. Biol. 38:7-23, 1984.

${ }^{56} \mathrm{McL}$ aren, A., and M. Buehr. Development of mouse germ cells in cultures of fetal gonads. Cell Differ. Dev. 31:185-195, 1990.

${ }^{57}$ McLaren, A., and D. Southee. Entry of mouse embryonic germ cells into meiosis. Dev. Biol. 187:107-113, 1997.

${ }^{58}$ McNatty, K. P., P. Smith, N. L. Hudson, D. A. Heath, D. J. Tisdall, et al. Development of the sheep ovary during fetal and early neonatal life and the effect of fecundity genes. J. Reprod. Fertil. Suppl. 49:123-135, 1995.

${ }^{59}$ Mochida, N., A. Akatani-Hasegawa, K. Saka, M. Ogino, Y. Hosoda, et al. Live births from isolated primary/early secondary follicles following a multistep culture without organ culture in mice. Reproduction 146:37-47, 2013.

${ }^{60}$ Moniruzzaman, M., R. M. Bao, H. Taketsuru, and T. Miyano. Development of vitrified porcine primordial follicles in xenografts. Theriogenology 72:280-288, 2009.

${ }^{61}$ Monk, M., M. Boubelik, and S. Lehnert. Temporal and regional changes in DNA methylation in the embryonic, extraembryonic and germ cell lineages during mouse embryo development. Development 99:371-382, 1987.

${ }^{62}$ Morohaku, K., Y. Hirao, and Y. Obata. Developmental competence of oocytes grown in vitro: has it peaked already? J. Reprod. Dev. 62:1-5, 2016.

${ }^{63}$ Morohaku, K., R. Tanimoto, K. Sasaki, R. KawaharaMiki, T. Kono, et al. Complete in vitro generation of fertile oocytes from mouse primordial germ cells. Proc. Natl. Acad. Sci. U.S.A. 113:9021-9026, 2016.

${ }^{64}$ Myers, M., F. H. Morgan, S. H. Liew, N. Zerafa, T. U. Gamage, et al. PUMA regulates germ cell loss and primordial follicle endowment in mice. Reproduction 148:211-219, 2014.

${ }^{65}$ Nagai, K., Y. Yanagawa, S. Katagiri, and M. Nagano. The relationship between antral follicle count in a bovine ovary and developmental competence of in vitro-grown oocytes derived from early antral follicles. Biomed. Res. (Tokyo, Japan) 37:63-71, 2016.

${ }^{66}$ O'Brien, M. J., J. K. Pendola, and J. J. Eppig. A revised protocol for in vitro development of mouse oocytes from primordial follicles dramatically improves their developmental competence. Biol. Reprod. 68:1682-1686, 2003.

${ }^{67}$ Obata, Y., T. Kono, and I. Hatada. Gene silencing: maturation of mouse fetal germ cells in vitro. Nature 418:497, 2002.

${ }^{68}$ Ohinata, Y., H. Ohta, M. Shigeta, K. Yamanaka, T. Wakayama, and M. Saitou. A signaling principle for the specification of the germ cell lineage in mice. Cell 137:571$584,2009$.

${ }^{69}$ Ohinata, Y., B. Payer, D. O'Carroll, K. Ancelin, Y. Ono, et al. Blimp1 is a critical determinant of the germ cell lineage in mice. Nature 436:207-213, 2005.

${ }^{70} \mathrm{Oi}$, A., H. Tasaki, Y. Munakata, K. Shirasuna, T. Kuwayama, and H. Iwata. Effects of reaggregated granulosa cells and oocytes derived from early antral follicles on the properties of oocytes grown in vitro. J. Reprod. Dev. 61:191-197, 2015.

${ }^{71}$ Oktay, K., H. Newton, J. Mullan, and R. G. Gosden. Development of human primordial follicles to antral 
stages in SCID/hpg mice stimulated with follicle stimulating hormone. Hum. Reprod. 13:1133-1138, 1998.

${ }^{72}$ Oktem, O., and B. Urman. Understanding follicle growth in vivo. Hum. Reprod. 25:2944-2954, 2010.

${ }^{73}$ Oulad-Abdelghani, M., P. Bouillet, D. Decimo, A. Gansmuller, S. Heyberger, et al. Characterization of a premeiotic germ cell-specific cytoplasmic protein encoded by Stra8, a novel retinoic acid-responsive gene. J. Cell Biol. 135:469-477, 1996.

${ }^{74}$ Pacchiarotti, J., C. Maki, T. Ramos, J. Marh, K. Howerton, et al. Differentiation potential of germ line stem cells derived from the postnatal mouse ovary. Differentiation 79:159-170, 2010.

${ }^{75}$ Park, E. S., and J. L. Tilly. Use of DEAD-box polypeptide-4 (Ddx4) gene promoter-driven fluorescent reporter mice to identify mitotically active germ cells in post-natal mouse ovaries. Mol. Hum. Reprod. 21:58-65, 2015.

${ }^{76}$ Pepling, M. E., and A. C. Spradling. Mouse ovarian germ cell cysts undergo programmed breakdown to form primordial follicles. Dev. Biol. 234:339-351, 2001.

${ }^{77}$ Pepling, M. E., E. A. Sundman, N. L. Patterson, G. W. Gephardt, L. Medico, Jr, and K. I. Wilson. Differences in oocyte development and estradiol sensitivity among mouse strains. Reproduction 139:349-357, 2010.

${ }^{78}$ Pesce, M., R. Canipari, G. L. Ferri, G. Siracusa, and M. De Felici. Pituitary adenylate cyclase-activating polypeptide (PACAP) stimulates adenylate cyclase and promotes proliferation of mouse primordial germ cells. Development 122:215-221, 1996.

${ }^{79}$ Picton, H. M. Activation of follicle development: the primordial follicle. Theriogenology 55:1193-1210, 2001.

${ }^{80}$ Rall, W. F., and G. M. Fahy. Ice-free cryopreservation of mouse embryos at -196 degrees $\mathrm{C}$ by vitrification. Nature 313:573-575, 1985.

${ }^{81}$ Resnick, J. L., L. S. Bixler, L. Cheng, and P. J. Donovan. Long-term proliferation of mouse primordial germ cells in culture. Nature 359:550-551, 1992.

${ }^{82}$ Robertson, J. F. ICI 182,780 (Fulvestrant)-the first oestrogen receptor down-regulator-current clinical data. Br. J. Cancer 85(Suppl 2):11-14, 2001.

${ }^{83}$ Saitou, M., S. C. Barton, and M. A. Surani. A molecular programme for the specification of germ cell fate in mice. Nature 418:293-300, 2002.

${ }^{84}$ Sakashita, A., Y. Kawabata, Y. Jincho, S. Tajima, S. Kumamoto, et al. Sex Specification and Heterogeneity of Primordial Germ Cells in Mice. PLoS ONE 10:e0144836, 2015.

${ }^{85}$ Sarraj, M. A., and A. E. Drummond. Mammalian foetal ovarian development: consequences for health and disease. Reproduction 143:151-163, 2012.

${ }^{86}$ Sasaki, K., S. Yokobayashi, T. Nakamura, I. Okamoto, Y. Yabuta, et al. Robust In Vitro Induction of Human Germ Cell Fate from Pluripotent Stem Cells. Cell Stem Cell 17:178-194, 2015.

${ }^{87}$ Sato, T., K. Katagiri, A. Gohbara, K. Inoue, N. Ogonuki, et al. In vitro production of functional sperm in cultured neonatal mouse testes. Nature 471:504-507, 2011.

${ }^{88}$ Seki, Y., K. Hayashi, K. Itoh, M. Mizugaki, M. Saitou, and Y. Matsui. Extensive and orderly reprogramming of genome-wide chromatin modifications associated with specification and early development of germ cells in mice. Dev. Biol. 278:440-458, 2005.

${ }^{89}$ Seki, Y., M. Yamaji, Y. Yabuta, M. Sano, M. Shigeta, et al. Cellular dynamics associated with the genome-wide epigenetic reprogramming in migrating primordial germ cells in mice. Development 134:2627-2638, 2007.

${ }^{90}$ Shen, W., L. Li, Z. Bai, Q. Pan, M. Ding, and H. Deng. In vitro development of mouse fetal germ cells into mature oocytes. Reproduction 134:223-231, 2007.

${ }^{91}$ Shen, W., D. Zhang, T. Qing, J. Cheng, Z. Bai, et al. Live offspring produced by mouse oocytes derived from premeiotic fetal germ cells. Biol. Reprod. 75:615-623, 2006.

${ }^{92}$ Smitz, J. E., and R. G. Cortvrindt. The earliest stages of folliculogenesis in vitro. Reproduction 123:185-202, 2002.

${ }^{93}$ Stott, D., and C. C. Wylie. Invasive behaviour of mouse primordial germ cells in vitro. J. Cell Sci. 86:133-144, 1986.

${ }^{94}$ Taketo, T., and S. S. Koide. In vitro development of testis and ovary from indifferent fetal mouse gonads. Dev. Biol. 84:61-66, 1981.

${ }^{95}$ Tam, P. P., and M. H. Snow. Proliferation and migration of primordial germ cells during compensatory growth in mouse embryos. J. Embryol. Exp. Morphol. 64:133-147, 1981.

${ }^{96}$ Telfer, E. E., and M. B. Zelinski. Ovarian follicle culture: advances and challenges for human and nonhuman primates. Fertil. Steril. 99:1523-1533, 2013.

${ }^{97}$ Wakeling, A. E., M. Dukes, and J. Bowler. A potent specific pure antiestrogen with clinical potential. Cancer Res. 51:3867-3873, 1991.

${ }^{98}$ Wandji, S. A., V. Srsen, P. W. Nathanielsz, J. J. Eppig, and J. E. Fortune. Initiation of growth of baboon primordial follicles in vitro. Hum. Reprod. 12:1993-2001, 1997.

${ }^{99}$ Wang, X., S. Catt, M. Pangestu, and P. Temple-Smith. Successful in vitro culture of pre-antral follicles derived from vitrified murine ovarian tissue: oocyte maturation, fertilization, and live births. Reproduction 141:183-191, 2011.

${ }^{100}$ Wang, C., E. R. Prossnitz, and S. K. Roy. Expression of G protein-coupled receptor 30 in the hamster ovary: differential regulation by gonadotropins and steroid hormones. Endocrinology 148:4853-4864, 2007.

${ }^{101}$ Wang, H., J. Xiang, W. Zhang, J. Li, Q. Wei, et al. Induction of germ cell-like cells from porcine induced pluripotent stem cells. Sci. Rep. 6:27256, 2016.

${ }^{102}$ Woods, D. C., and J. L. Tilly. Isolation, characterization and propagation of mitotically active germ cells from adult mouse and human ovaries. Nat. Protoc. 8:966-988, 2013.

${ }^{103}$ Xiao, S., J. Zhang, M. M. Romero, K. N. Smith, L. D. Shea, and T. K. Woodruff. In vitro follicle growth supports human oocyte meiotic maturation. Sci. Rep. 5:17323, 2015.

${ }^{104}$ Yamaji, M., Y. Seki, K. Kurimoto, Y. Yabuta, M. Yuasa, et al. Critical function of Prdm14 for the establishment of the germ cell lineage in mice. Nat. Genet. 40:1016-1022, 2008.

${ }^{105}$ Yang, M. Y., and J. E. Fortune. The capacity of primordial follicles in fetal bovine ovaries to initiate growth in vitro develops during mid-gestation and is associated with meiotic arrest of oocytes. Biol. Reprod. 78:1153-1161, 2008.

${ }^{106}$ Yang, X., C. Kubota, H. Suzuki, M. Taneja, P. E. Bols, and G. A. Presicce. Control of oocyte maturation in cows-biological factors. Theriogenology 49:471-482, 1998. 
${ }^{107}$ Yoshimizu, T., M. Obinata, and Y. Matsui. Stage-specific tissue and cell interactions play key roles in mouse germ cell specification. Development 128:481-490, 2001.

${ }^{108}$ Zarate-Garcia, L., S. I. Lane, J. A. Merriman, and K. T. Jones. FACS-sorted putative oogonial stem cells from the ovary are neither DDX4-positive nor germ cells. Sci. Rep. 6:27991, 2016

${ }^{109}$ Zhang, Z. P., G. J. Liang, X. F. Zhang, G. L. Zhang, H. $\mathrm{H}$. Chao, et al. Growth of mouse oocytes to maturity from premeiotic germ cells in vitro. PLoS ONE 7:e41771, 2012.
${ }^{110}$ Zsebo, K. M., D. A. Williams, E. N. Geissler, V. C. Broudy, F. H. Martin, et al. Stem cell factor is encoded at the Sl locus of the mouse and is the ligand for the c-kit tyrosine kinase receptor. Cell 63:213-224, 1990.

${ }^{111}$ Zsebo, K. M., J. Wypych, I. K. McNiece, H. S. Lu, K. A. Smith, et al. Identification, purification, and biological characterization of hematopoietic stem cell factor from buffalo rat liver-conditioned medium. Cell 63:195-201, 1990. 